\title{
Immune-inspired Evolutionary Algorithm for Constrained Optimization
}

\author{
Weiwei Zhang ${ }^{1,2}$ and Gary G. Yen ${ }^{2}$ \\ ${ }^{1}$ Department of Computer Science, Chongqing University, Chongqing 40044, PR CHINA \\ ${ }^{2}$ School of Electrical and Computer Engineering, Oklahoma State University, Stillwater, OK-74078, USA
}

\begin{abstract}
This paper proposes an artificial immune system based algorithm for solving constrained optimization problems, inspired by the principle of the vertebrate immune system. The analogy between the mechanism of vertebrate immune system and constrained optimization formulation is first given. The population is divided into two groups- feasible individuals and infeasible individuals. The infeasible individuals are viewed as the inactivated immune cells approaching the feasible regions by decreasing the constraint violations whereas the feasible individuals are treated as activated immune cells searching for the optima. The interaction between them through the extracted directional information is facilitated mimicking the functionality of $T$ cells. This mechanism not only encourages infeasible individuals approaching feasibility regions, but facilitates exploring the boundary between the feasible and infeasible regions in which optima are often located. This approach is validated and performance is quantified by the benchmark functions used in related researches through statistical means with those of the state-of-the-art from various branches of evolutionary computation paradigms. The performance obtained is fairly competitive and in some cases even better.
\end{abstract}

Index Terms-Artificial immune system, constrained optimization, constraint handling

\section{INTRODUCTION}

$\mathrm{A}$ rtificial immune system (AIS) is a newly emerging computational paradigm inspired by the fundamentals of immune system.

\subsection{Biological Immune System}

In biology, the human or other vertebrate immune system is an adaptive, distributed, robust, self-regulate, and complicated system which constituted by cells, molecules, and organs that protect the body against known and unknown diseases. The immune system possesses multilevel defense mechanisms. The protection layers such as physical barriers and innate immune system constitute the non-specific response, whereas the adaptive immune system is responsible for the specific response. Pathogens or any molecules that stimulate the immune response are simply called antigens in this paper. Antigens in the environment are blocked from getting into the body by physical barriers, such as skin and hairs, at first. Underneath the skin, there are physiological barriers such as saliva and stomach acids which can kill most microorganisms ingested in food and water. Antigens that escape from the physical barriers are tackled by the other non-specific response mechanism called innate immune system. Innate immune cells such as macrophages can recognize foreign antigens and fragment them into peptides. These peptides, express on the surface of macrophage, make it serve as antigen present cells. Meanwhile, these antigen present cells secrete activating molecules such as chemical signals to stimulate the start of the adaptive immune response. The adaptive immune response is an acquired ability that reacts specifically to the attacking antigens. The adaptive immune system is composed of two types of lymphocytes: $\mathrm{B}$ lymphocytes (B-cells) and $\mathrm{T}$ lymphocytes (T-cells). The main functions of B-cells include the production and secretion of antibodies as a response to bind with antigens. Each B-cell is programmed to produce a specific antibody. The production of binding antibodies is usually a way of signaling other cells, such as scavenging cells, to remove the bound substance. The main functions of T-cells include the regulation of other cells' actions and directly attack the host infected cells. T-cells work, primarily, by secreting cytokines, lymphokines, or the other chemical substance to maintain the regular function of the immune system. Moreover, the adaptive response is also able to memorize the attacking antigens so that it will be effectively fought against the same or similar antigens in the future [1-3].

\subsection{AIS for Constrained Optimization}

Normally, immune cells patrol the body circulating along the blood flow to detect if there are antigens present. Once antigen is detected, the immune response is stimulated. The activated immune cells eliminate the antigens directly or secrete signals to recruit other immune cells to help. Immune cells are supposed to be able to recognize the antigen pattern, extract the antigen feature, learn the antigen type, select the suitable reacting mechanism to control the harm, and remember the experience. To make it simpler, due to the B-cells having the capability of antigen representation as same as the innate immune cells, let us omit the innate immune system and only consider the immune cells in adaptive immune system. Naïve B cells patrol the body searching 
for the antigen. Without activation of the chemical signals, naïve B cells do not perform specific immune response. However, with the irritation of chemical signals secreted by T-cells, naïve B cells transform into effector B cells, modify their antibody, and fight with the antigens. In spirit, chemical signals work as constrained conditions which control the function of B-cells in the immune response. Similarly in many real world problems, the decision variables are subject to constraints which limit the search for optimal solution only in the feasible region. A constrained optimization problem can be defined as follows,

subject to:

$$
\text { Minimize } f(x)
$$

$$
\begin{aligned}
& g_{i}(x) \leq 0, i=1, \cdots, q \\
& h_{i}(x)=0, i=q+1, \cdots, m
\end{aligned}
$$

where a vector of decision variables $x=\left[x^{1}, \cdots, x^{n}\right]$ in which each $x^{j}, j=1, \cdots, n$ is bounded by a lower and a upper bound, $L^{j} \leq x^{j} \leq U^{j}$ defining the search space $S \subseteq \mathfrak{R}^{n}, q$ is the number of inequality constraints while $m-q$ is the number of equality constraints. A solution satisfies all the inequality and equality constraints is called a feasible solution, whereas a solution violates at least one constraint is called infeasible solution. The inequality constraints that satisfy $g_{i}(x)=0$ at the global optimum solution are called active constraints.

In this article, a novel constrained artificial immune algorithm is proposed based on immune response principle. A new perspective from the signal transfer and the interaction among the immune cells is proposed to handle the constrained condition and optimize the problems. The remainder of this paper is organized as follows. Section II reviews related works of handling constrained optimization problems using AIS. In Section III, the proposed algorithm is presented in detail. Section IV discusses the results obtained for some selected benchmark functions and compares the results obtained by the states-of-the-art. Finally, Section V presents some concluding remarks and relevant observations.

\section{LITERATURE REVIEW}

Equipped with fundamental characteristics of biological immune system, AIS has received appreciable attention to solving complex computational problems in the past few years. However, only very few research work exists in the field of constraint handling using AIS. A survey paper which summarized algorithms ever proposed in handing constrained optimization based on artificial immune system can be found in [4].

\subsection{Hybrid AIS with GA and Other Mechanisms}

A branch of hybrid AISs with GA was proposed [5-8]. The hybrid algorithms possess two layers: GA outer layer and AIS inner layer. The outer GA is responsible for the unconstrained objective function optimization, while the inner AIS embedded into the outer GA helps population approaching feasible regions.

Based on its constraint-handling technique, a clonal selection evolutionary strategy was proposed [9]. Immune algorithm is also integrated with hill climbing local search in [10], and integrated with particle swarm optimization (PSO) in [11] for constrained optimization problems.

In all above designs, AISs play a complementary role in either handling the constraints or improving the search ability. Although some of these hybrid algorithms show very competitive results, the hybrid schema often adversely increase the complexity of the designs.

\subsection{AIS based Constraint Handling Algorithms}

AIS algorithms that are exclusively based on process and principles directly extracted from the biological immune system were proposed [12-18].

\subsubsection{AIS based on clonal selection algorithm}

Cruz-Cortés et al. [12] proposed an approach based on CLONALG [19] to constraint handling using AIS. Gaussian-distributed mutations, Cauchy-distributed mutations, and controlled uniform mutation are compared. In [13], the feasible individuals and infeasible individuals are given different mutation operations. The mutation mechanism is efficient to balance the exploration and exploitation during the evolution of search. The algorithm is simple to implement and the experiment result is found better than that of [12].

As these algorithms applying the unconstrained optimization technique to the constraint optimization, it is hard to solve the problems with discontinuous search space or multiple disconnected feasible regions.

\subsubsection{AIS based on idiotypic network approach}

A new algorithm which combines clonal selection theory with idiotypic network theory was developed by $\mathrm{Wu}$ [14-15]. In the paper, idiotypic network selection operator is used to control the number of good solutions; somatic hypermutation and receptor editing operators are constructed to explore the search space; and bone marrow operator is to maintain the diversity of the solutions. Adaptive penalty function is introduced to transform the constrained optimization problem into an unconstrained one. The simulation results show that the algorithm is effective and efficient.

\subsubsection{AIS based on T-cell model}

In [16-17], T-cells model to handle the constrained optimization was proposed. Based on the maturation or development level, T-cells are divided into three groups: Virgin cells (VC), Effector cells (EC), and Memory cells (MC). Giving each group a specific size of population, mutation operator, and selection principles, the algorithm adaptively adjusts the mechanism under different 
conditions. From the experiment comparison, T-cells model is proven to be very competitive with respect to the state-of-the-art designs. The algorithm is novel and effective, but it is complicated in the structure. Many design parameters requiring additional tuning may limit its uses in applications.

\subsection{AIS with Special Characteristics}

An algorithm deployed the communication between innate immune response and adaptive response in immune system was proposed in [18]. Vaccine operator proposed in [20] is used to explore the search area. $10 \%$ of the antibodies from antibody population are selected to form Major Histocompatibility Complex (MHC) as the direction extracted from the better individuals. The combination of the directed mutation with vaccines extracted from the early stage of the algorithm give a speedy way of discovering the feasible regions. The performance of the algorithm is very competitive, and the vaccine operator guarantees the high feasible success rate. However, high computational complexity is needed to guarantee the extracted vaccines are able to help antibodies traverse the whole searching space without exploring the same area repeatedly.

The optima-searching ability of AIS has been proven to be effective in constrained optimization problems, but the constraint-handling mechanism remains deficient. The interrelation among the system's components is rarely mentioned.

\section{PROPOSED ALGORITHM}

The immune response of B-cells to an antigen is a process of pursuing the best binding antibody to the antigen which is considered an optimization process. Naïve B-cells circulate in the body searching for the infectious spot as global search, while effector B-cells modify their shapes to match the antigen as local search. T-cells balance the global and local search through secreting chemical signals. Additionally, the chemical signals secreted by T-cells perform exactly as the constraints to control the function of B-cells. Without the stimulating signal, B-cells only respond to the certain antigen in the manner of innate immune response in a nonspecific way. When B-cells receive the stimulating signals, the activated B-cells will adaptively adjust themselves to react to the antigen in a specific manner. According to the different satisfactions to the constraints, B-cells are separated into two distinct types- naive (inactivated) B-cells and effector (activated) B-cells. The way B-cells under the control of T-cells in pursuing the best binding antibody to the antigen can be considered as a constrained optimization process. Inspired by the effective and rapid response of B-cells to antigens, an AIS algorithm is proposed herein. Constraints separate the candidate solutions into two groups- infeasible and feasible which nicely match the two states of B-cells. Infeasible individuals resemble the naïve B-cells to explore the whole search space while feasible individuals mimic the effector B-cells to locate the exact antigen (optima). Chemical signals play an intermediate role of transferring the information between infeasible and feasible individuals and balance the exploration and exploitation.

The proposed algorithm as shown in Fig. 1 depicts the flowchart as how the algorithm works.

1) Randomly initialize $P$ individuals in the decision space $x_{i}, i=1, \cdots, P$, where $x_{i}$ is the $i$ th individual in the $n$-dimensional decision space.

2) Evaluate the constraint violation $v\left(x_{i}\right)$ for each individual in the population as

$v\left(x_{i}\right)=\sum_{j=1}^{q} \max \left(0, g_{j}\left(x_{i}\right)\right)+\sum_{j=q+1}^{m} \max \left(0,\left|h_{j}\left(x_{i}\right)\right|-\delta\right)$

where $\delta$ is the tolerance value that help transform the equality constraint into an inequality one.

3) Categorize the population into two distinct groups: if $v\left(x_{i}\right)>0$, add $x_{i}$ into infeasible group, IF . Otherwise, add it into feasible group, $F$.

4) If feasible group $F$ is not empty, evaluate the fitness value $f\left(x_{r}\right)$ for each feasible individual $x_{r} \in F, r=1, \cdots,|F|,|\cdot|$ indicates the cardinality. Then sort the individuals in the ascending order according to their fitness values (assuming minimization problem).

a) Cloning: For each feasible individual $x_{r}$ in the ranking order of $r$, asexually produce $n c\left(x_{r}\right)$ clones

$n c\left(x_{r}\right)=\operatorname{round}\left(\beta \frac{P}{r}\right), \forall x_{r} \in F$

where $\beta$ is a multiplying factor (i.e., usually $0.1)$, round $(\cdot)$ denotes the rounding operation to the closest integer. Since all the individuals are sorted in the ascending order previously, the individual with higher objective value will receive more clones.

b) Hypermutation: Hypermutate each clone at a rate inversely proportional to their affinity using

$$
\begin{aligned}
& f^{*}\left(x_{r}\right)=\frac{f\left(x_{r}\right)}{\max _{x_{r} \in F} f\left(x_{r}\right)}, \\
& \alpha\left(x_{r}\right)=\exp \left(-\rho f *\left(x_{r}\right)\right), \\
& x_{r}^{\prime}=x_{r}+\alpha\left(x_{r}\right) \times N(0,1) \times \frac{\text { maxgen }- \text { gen }}{\text { maxgen }},
\end{aligned}
$$

where $N(0,1)$ is a Gaussian random number with zero mean and unit variance, $f *\left(x_{r}\right)$ is the normalized fitness of $x_{r}, \rho$ is the decay constant that controls the step size of the mutation (default value is set to 5), maxgen is the 
maximum number of generations, and gen refers to the current generation.

c) Selection: Evaluate the affinity of the clones. If the best individual of the set of mutated clones has the higher affinity (lower objective value) than the original one, substitute it. Otherwise, keep the original individual.

d) Add the newly generated infeasible clones into the infeasible group, IF .

5) For each individual $x_{s} \in I F, \quad s=1, \cdots,|I F|$ with their constraint violation $v\left(x_{s}\right)$, perform the following operations.

a) Direction extraction: if there are feasible individuals exist in the population, choose a feasible individual $x^{*}$ by roulette wheel selection based on their fitness values, and extract the direction information $d r$ as follows,

$$
d r=\frac{x_{s}-x^{*}}{\left\|x_{s}-x^{*}\right\|} .
$$

Otherwise, $10 \%$ of the best infeasible individuals are chosen to generate the direction based on their constraint violations.

b) Location update: Infeasible individuals update their location either by the way of the extracted direction $d r$,

$x_{s}^{\prime}=x_{s}+\omega \times d r \times U(0,1)$,

or just choose a random velocity to move

$$
x_{s}^{\prime}=x_{s}+N(0, \sigma) \text {, }
$$

where $\omega \in[0,1]$ is an user defined parameter controlling the effect of the direction information, ( $\omega=0.1$ as suggested) $U(0,1)$ is a uniform random number generated between 0 and 1 , Gaussian random number is used to introduce the random velocity. $\sigma$ controls the magnitude of the velocity (the default value is 0.2 ). To further improve upon the exploration, the location update repeats a few times and the best position is chosen to replace the current one. During implementation, either (8) or (9) can be chosen to update the individuals' location equally or bias one of them based on the performance. The former one is applied in the paper.

6) Suppression: Combine the infeasible and feasible groups together, and delete the similar individuals using a predefined suprresion threshold (problem dependent, 0.5 for reference).

7) Receptor editing: The worst $20 \%$ individuals of the whole population are substituted at random (binary tournament selection is adopted here, few candidates are generated randomly, the one in the most sparse area is chosen).

8) If stopping condition is not met go to Step 2. Otherwise, output the best one (in term of affinity) in the feasible group.

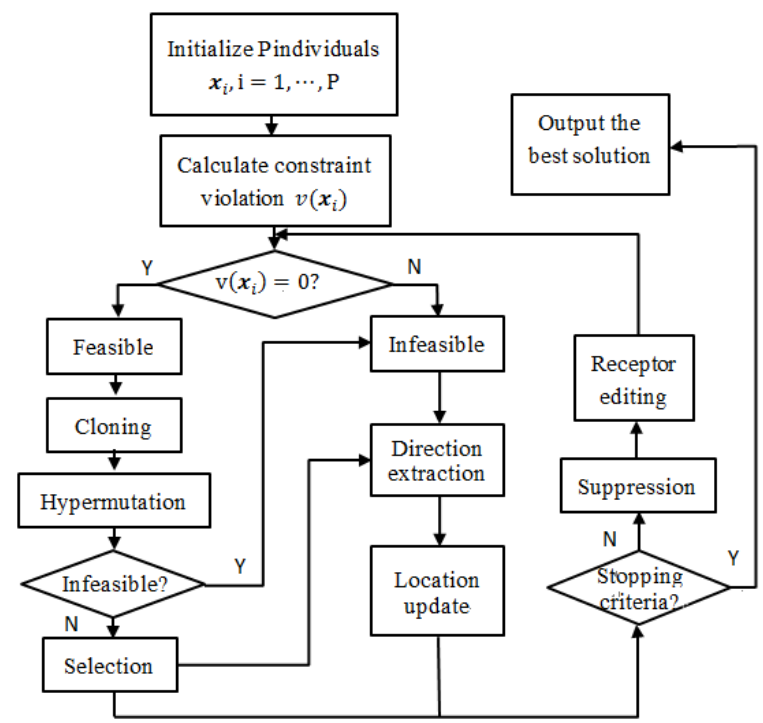

Fig. 1 Flowchart of the proposed algorithm

Since feasible and infeasible individuals take different responsibility in handling constrained optimization problems, we divide them into different groups and facilitate the interaction between them in the proposed algorithm. The function of feasible individuals is to search for the optima in the feasible regions, which is regarded as an unconstrained optimization search. Considering the fairly good performance of the representative algorithm in clonal selection theoryCLONALG [19] for unconstrained multimodal optimization, we borrow the operators for optimization in feasible regions here. The individual with higher affinity will bear more clones and lower mutation rate, which give more chance to the regions with higher objective value and encourage the local search. Moreover, the iteration is considered as a parameter to control the convergence of the algorithm. With the increase of generation count, the step size of hypermutation will become smaller and smaller. This implies a larger mutation step at the beginning and becomes smaller and smaller toward the end of the search process.

On the other hand, constraints may restrict the feasible search space into small and disconnected regions Our purpose is to search for these feasible regions and then locate the optima. So the task of infeasible individual is on one hand, global search, to explore the whole search space and avoid to get stuck in the local optima. On the other hand, approach the feasible regions 
as soon as possible. The location update operator is introduced to relocate the infeasible individuals. In addition to guide infeasible individual moving along the direction in reduction of the constraint violation, two choices are offered to each of them. Extracted directions are used to accelerate the infeasible individuals access the feasible regions, while random velocity is used to maintain the diversity. When there is no feasible individual found, a proportion of the best infeasible individuals are chosen to guide the rest of the individuals which accelerate in finding the feasible regions. Otherwise, direction information is extracted from feasible individuals to guide the infeasible individuals. Once some individuals reach the feasible regions, moving in the direction to these feasible individuals will accelerate the infeasible individuals to approach the nearest feasible regions. But, this may also attract all individuals move to the few early discovered feasible regions and stuck in these regions forever. Then, the algorithm will lose the ability of finding the other disconnected feasible regions and eventually lead to the premature convergence. Therefore, some of the infeasible individuals update their locations randomly.

Suppression and Receptor editing are used to delete the crowed and similar individuals and make space for the newly generated individuals to maintain the diversity. Tournament selection used in receptor editing is capable of retaining the best individuals and at the same time balancing the size of feasible and infeasible groups.

\section{EXPERIMENTAL RESULTS AND DISCUSSIONS}

To examine the performance quantitatively, we applied the proposed algorithm to the constrained benchmark functions available from [21]. These benchmark functions consist of linear, nonlinear, quadratic, and cubic functions. Some of these functions have high dimensional decision space and very low feasibility ratio. Manyconstraints involved are equality constraints. For most of these testing functions, it is not easy even locating a feasible region.

Each benchmark problem is run for 30 independent trials. Best (b), worst (w), mean (Mn), standard deviation(sd), and success rate (FR) to converging to a feasible solution are given to compare with the other algorithms, which are abbreviated as b, w, Mn, Md, sd, and FR in the tables, respectively. All the statistical results were taken only with respect to the runs in which a feasible solution was reached at the end.

All the equality constraints are transformed into inequality constraint by using a tolerance factor $\delta$, $|h(x)|-\delta \leq 0(\delta=0.0001$ as suggested $)$. To deal with equality constraints, dynamic mechanism originally proposed in [22] is adopted here.

35,000 objective function evaluations are applied as a stopping criterion for each test problem. The statistical results are summarized in Table I. From Table I, we can easily find that the proposed algorithm shows very promising performance in handling these constrained problems. The algorithm achieves the required accuracy level for G01, G03, G06, G08, G11, G12, and G13. Moreover, it attains the best result for G13, even better than any known best result. It is worthy to note that all 30 runs for 13 test problems produce $100 \%$ success rate in converging to a feasible solution. In addition, the stochastic ranking algorithm (SR) [23], GA-AIS ${ }^{c}$ [24], AIS [12], AIS $_{\text {const }}$ [13], and T-cell algorithm [17] as the reference are chosen to compare with the proposed algorithm. To make the comparison less complicated, only the best and mean results are considered here to judge the performance of the algorithms. GA-AIS ${ }^{\mathrm{c}}$ [24], AIS [12], AIS $_{\text {const }}$ [13], and T-cell algorithm [17] are the typical algorithms proposed for constrained optimization based on AIS. The proposed algorithm has a better or equal performance when compare with GA-AIS ${ }^{\mathrm{c}}$ [24] in almost all the test problems except G06 and G10. Even for G06 and G10, only mean values of GA-AIS ${ }^{c}$ are slightly better than that of the proposed algorithm. Best values of the proposed algorithm outperform GA-AIS ${ }^{\mathrm{c}}$ in all the test problems. To compare with the AIS [12] and AIS $_{\text {const }}$ [13], the results are similar. AIS [12] is equal to or outperformed by the proposed algorithm except for

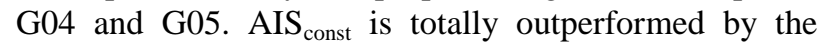
proposed algorithm. T-cell algorithm obtains very competitive results. T-cell algorithm is better than the proposed algorithm in G04, G06, and G09, and has a better best value in G07 and G10. However, the proposed algorithm acts better or equal to T-cell algorithm in the rest of 8 test problems (i.e., G01, G02, G03, G05, G08, G11, G12, and G13). According to the comparison, the proposed algorithm is better than or equal to these state-of-the-art AIS designs in most of the 13 test problems.

Stochastic ranking algorithm (SR) [23], a well-regarded evolutionary design for constrained optimization, is also chosen for comparison with the proposed algorithm. From the Table I, SR outperforms the proposed algorithm in five test problems (i.e., G02, G04, G05, G07, and G09). Nonetheless, the proposed algorithm outperforms SR also in five test problems (i.e., G03, G06, G10, G11, and G13). Although their performances favor different test problems, the difference is quite small. On the whole, the proposed algorithm is as competitive as the other AIS-based algorithm and the state of the art. It needs to be mentioned that, for some problems, fitness evaluation of the infeasible region is not applicable, then the algorithm as SR which based on the evaluation of the infeasible solution will become useless.

Through the results comparison, the proposed algorithm is found fairly competitive with respect to some chosen state-of-the-art designs in the constrained optimization field. 


\section{CONCLUSIONS}

Inspired by the fundamentals of biological immune system, a novel artificial immune system is proposed to solve constrained optimization in this paper. We exploit the immune response from the perspective of information transfer and encourage the interactions between feasible and infeasible individuals. The direction information extracted is used to guide the infeasible individuals effectively and efficiently toward the nearby feasible regions. These infeasible individuals near the boundary of feasible and infeasible regions are reused to search the boundary thoroughly. Local search operator of feasible individuals is borrowed from the traditional clonal selection algorithm which had been validated to be effective in handling the unconstrained optimization. The global search of infeasible individuals simply employs the Gaussian random variable to introduce the disturbance. The experimental results show the performance of the proposed algorithm in solving 13 frequently used benchmark functions. Through comparing with the selected representative algorithms from various branches of evolutionary computation paradigms, the proposed algorithm is confirmed to be competitive.

\section{ACKNOWLEDGEMENTS}

This research is funded by the Project No. CDJXS10181131, supported by the Fundamental Research Funds for the Central Universities.

\section{REFERENCES}

[1] L. N. de Castro and J. Timmis, Artificial Immune System: A New Computational Intelligence Approach, SpringerVerlag: London, UK, 1996.

[2] D. Dasgupta, Artificial Immune Systems and Their Application, Springer-Verlag: Heidelberg, Germany, 1999.

[3] AISWeb The online home of artificial immune systems http://www.artificial-immune-systems.org.

[4] N. Cruz-Cortes, "Handling constraints in global optimization using artificial immune systems: a survey," Studies in Computational Intelligence, vol. 198, pp. 237-262, 2009.

[5] P. Hajela and J.S. Yoo, "Immune network modeling in design optimization," In D. Corne, M. Dorigo, and F. Glover, editors, New Ideas in Optimization, McGraw-Hill: New York, NY, pp. 203-215, 1999.

[6] C.A. Coello Coello and N. Cruz-Cortes, "Hybridizing a genetic algorithm with an artificial immune system for global optimization," Engineering Optimization, vol. 36, pp. 607-634, 2004.

[7] H.S. Bernardino, H.J. Barbosa, and A.C.C. Lemonge, "A hybrid genetic algorithm for constrained optimization problems in mechanical engineering," Proceedings of IEEE Congress on Evolutionary Computation, Singapore, pp. 646-653, 2007.
[8] H.S. Bernardino, H.J. Barbosa, A.C.C. Lemonge, and L.g. Fonseca, "A new hybrid AIS-GA for constrained optimization problems in mechanical engineering," Proceedings of IEEE Congress on Evolutionary Computation, Hong Kong, China, pp. 1455-1462, 2008.

[9] W.P. Ma, L.C. Jiao, M.G. Gong, and R.H. Shang, "Immune clonal selection evolutionary strategy for constrained optimization," Proceedings of Pacific Rim International Conference on Artificial Intelligence, Guilin, China, pp. 661-670, 2006.

[10] A.R. Y1ldiz, "A novel hybrid immune algorithm for global optimization in design and manufacturing," Robotics and Computer-Integrated Manufacturing, vol. 25, pp. 261-270, 2009.

[11] A.J. Ouyang, "A hybrid immune PSO for constrained optimization problems," Proceedings of IEEE International Conference on Bio-Inspired Computing: Theories and Applications, Changsha, China, pp. 1291-1295, 2010.

[12] N. Cruz-Cortes, D. Trejo-Perez, and C.A. Coello Coello, "Handling constraints in global optimization using an artificial immune system," In C. Jacob, M.L. Pilat, P. J. Bentley, and J.I. Timmis, Editors, ICARIS 2005. LNCS, vol. 3627, pp. 234-247, 2005.

[13] V.S. Aragon, S.C. Esquivel, and C.A. Coello Coello, "Artificial immune system for solving constrained optimization problems," Revista Iberoamericana de Inteligencia Artificial, vol. 35, pp. 55-66, 2007.

[14] J.Y. Wu, "Artificial immune system for solving constrained global optimization problems," Proceedings of IEEE Symposium on Artificial Life, Honolulu, HI, pp. 92-99, 2007.

[15] J.Y. Wu, "Solving constrained global optimization via artificial immune system," International Journal on Artificial Intelligence Tools, vol. 20, pp. 1-27, 2011.

[16] V.S. Aragon, S.C. Esquivel, and C.A. Coello Coello, "A novel model of artificial immune system for solving constrained optimization problems with dynamic tolerance factor," Proceedings of Mexico International Conference on Artificial Intelligence, Aguascalientes, Mexico, pp.19-29, 2007.

[17] V.S. Aragon, S.C. Esquivel, and C.A. Coello Coello, "A modified version of a T-cell Algorithm for constrained optimization problem," International Journal for Numerical Method in Engineering, vol. 84, pp. 351-378, 2010.

[18] K.M. Woldemariam and G.G. Yen, "Constrained optimization using artificial immune system," Proceedings of IEEE Congress on Evolutionary Computation, Barcelona, Spain, pp. 1-8, 2010.

[19] L.N. de Castro and F. J. Von Zuben, "Learning and optimization using the clonal selection principle," IEEE Transactions on Evolutionary Computation, vol. 6, pp. 239-251, 2002.

[20] K.M. Woldemariam and G.G Yen, "Vaccine-enhanced artificial immune system for multimodal function optimization," IEEE Transactions on Systems, Man, and Cybernetics, Part B, vol. 40, pp. 218-228, 2010. 
[21] J.J. Liang, T.P. Runarsson, E. Mezura-Montes, M. Clerc, P.N. Suganthan, C. A. Coello Coello, and K. Deb, "Problem definitions and evaluation criteria for the CEC 2006 special session on constrained real-parameter optimization," Proceedings of IEEE Congress on Evolutionary Computation, Vancouver, Canada, 2006.

[22] S.B. Hamida and M. Schoenauer, "ASCHEA: new results using adaptive segregational constraint handling," Proceedings of IEEE Congress on Evolutionary Computation, Honololu, HI, pp. 82-87, 2002.
[23] T.P. Runarsson and X. Yao, "Stochastic ranking for constrained evolutionary optimization," IEEE Transactions on Evolutionary Computation, vol. 4, pp 284-294, 2000.

[24] H.S. Bernardino, H.J.C. Barbosa, A.C.C. Lemonge, and L.G. Fonseca, "On GA-AIS hybrids for constrained optimization problems in engineering, constraint-handling in evolutionary optimization," Studies in Computational Intelligence, vol. 198, pp. 167-192, 2009.

TABLE I.

COMPARISON OF EXPERIMENT RESULTS ON BENCHMARK FUNCTIONS IN [38]

\begin{tabular}{|c|c|c|c|c|c|c|c|}
\hline Function & & SR [23] & GA- AIS $^{\mathrm{c}}[24]$ & AIS [12] & $\mathrm{AIS}_{\text {const }}[13]$ & T-cell [17] & Proposed AIS \\
\hline \multirow{5}{*}{$\begin{array}{c}\text { G01 } \\
-15.0000\end{array}$} & $\mathrm{~b}$ & -15.000 & -14.98887 & -14.9874 & -14.993 & -15 & -15.0000 \\
\hline & Mn & -15.000 & -14.97514 & -14.7264 & -14.989 & -15 & -15.0000 \\
\hline & w & -15.000 & -14.93905 & -12.9171 & -14.982 & -15 & -15.0000 \\
\hline & sd & $0.0 \mathrm{E}+00$ & 8.06E-03 & $6.07 \mathrm{E}-01$ & $2.98 \mathrm{E}-03$ & 0.0 & $1.0 \mathrm{E}-08$ \\
\hline & FR & $100 \%$ & $100 \%$ & $100 \%$ & $100 \%$ & $100 \%$ & $100 \%$ \\
\hline \multirow{5}{*}{$\begin{array}{c}\text { G02 } \\
-0.8036191\end{array}$} & $\mathrm{~b}$ & -0.803515 & -0.7834507 & -0.8017 & -0.7821 & -0.801367 & -0.8033658 \\
\hline & Mn & -0.781975 & -0.7351923 & -0.7434 & -0.7573 & -0.752975 & -0.78142542 \\
\hline & w & -0.726288 & -0.6567412 & -0.6268 & -0.7230 & -0.687827 & -0.72769313 \\
\hline & $\mathrm{sd}$ & $2.0 \mathrm{E}-02$ & $2.26 \mathrm{E}-02$ & 4.14E-02 & $-1.4 \mathrm{E}-02$ & $3.20 \mathrm{E}-02$ & $5.19 \mathrm{E}-03$ \\
\hline & FR & $100 \%$ & $100 \%$ & $100 \%$ & $100 \%$ & $100 \%$ & $100 \%$ \\
\hline \multirow{5}{*}{$\begin{array}{c}\text { G03 } \\
-1.0005\end{array}$} & $\mathrm{~b}$ & -1.000 & -0.8879961 & -1.0 & -1.0 & -1.0 & -1.0005 \\
\hline & Mn & -1.000 & -0.4908986 & -1.0 & -0.9880 & -1.0 & -1.0005 \\
\hline & w & -1.000 & -0.0406379 & -1.0 & -0.9108 & -1.0 & -1.0005 \\
\hline & $\mathrm{sd}$ & $1.9 \mathrm{E}-04$ & $2.12 \mathrm{E}-02$ & 0.0 & $2.50 \mathrm{E}-02$ & 0.0 & $1.77 \mathrm{E}-05$ \\
\hline & FR & $100 \%$ & $100 \%$ & $100 \%$ & $100 \%$ & $100 \%$ & $100 \%$ \\
\hline \multirow{5}{*}{$\begin{array}{c}\text { G04 } \\
-30665.5387\end{array}$} & $\mathrm{~b}$ & -30665.539 & -30665.3889 & -30665.5387 & -30665.1117 & -30665.5385 & -30665.5377 \\
\hline & Mn & -30665.539 & -30662.9301 & -30665.5386 & -30645.9122 & -30665.5384 & -30665.4766 \\
\hline & w & -30665.539 & -30665.5270 & -30665.5386 & -30553.7827 & -30665.5382 & -30665.4057 \\
\hline & $\mathrm{sd}$ & $2.0 \mathrm{E}-05$ & 2.28 & $0.0 \mathrm{E}+00$ & $3.19 \mathrm{E}+01$ & $1.0 \mathrm{E}-04$ & 8.69E-03 \\
\hline & FR & $100 \%$ & $100 \%$ & $100 \%$ & $100 \%$ & $100 \%$ & $100 \%$ \\
\hline \multirow{5}{*}{$\begin{array}{c}\text { G05 } \\
5126.4967\end{array}$} & $\mathrm{~b}$ & 5126.497 & 5166.0885 & 5126.999 & 5126.660 & 5126.6255 & 5126.5176 \\
\hline & $\mathrm{Mn}$ & 5128.881 & 5204.1544 & 5436.1278 & 5468.743 & 5378.2678 & 5241.0371 \\
\hline & w & 5142.472 & 5256.9017 & 6111.1714 & 6112.072 & 6112.1181 & 5800.9546 \\
\hline & $\mathrm{sd}$ & $3.5 \mathrm{E}+00$ & $3.85 \mathrm{E}+01$ & $3.0 \mathrm{E}+02$ & $3.39 \mathrm{E}+02$ & $2.98 \mathrm{E}+02$ & $1.7 \mathrm{E}+02$ \\
\hline & FR & $100 \%$ & $12 \%$ & $90 \%$ & $75 \%$ & $80 \%$ & $100 \%$ \\
\hline \multirow{5}{*}{$\begin{array}{c}\text { G06 } \\
-6961.81385\end{array}$} & $\mathrm{~b}$ & -6961.814 & -6961.7961 & -6961.8105 & -6961.7940 & -6961.81387 & -6961.81385 \\
\hline & $\mathrm{Mn}$ & -6875.940 & -6961.7659 & -6961.8065 & -6960.3768 & -6961.81386 & -6961.76486 \\
\hline & w & -6350.262 & -6961.7558 & -6961.7981 & -6956.7421 & -6961.81385 & -6961.73297 \\
\hline & sd & $1.6 \mathrm{E}+02$ & $1.23 \mathrm{E}-02$ & $2.7 \mathrm{E}-03$ & $1.18 \mathrm{E}+00$ & $3.9 \mathrm{E}-05$ & $1.9 \mathrm{E}-02$ \\
\hline & FR & $66 \%$ & $100 \%$ & $100 \%$ & $100 \%$ & $100 \%$ & $100 \%$ \\
\hline
\end{tabular}




\begin{tabular}{|c|c|c|c|c|c|c|c|}
\hline G07 & $\mathrm{b}$ & 24.307 & 24.4482393 & 24.5059 & 24.531708 & 24.3209 & 24.3340080 \\
\hline \multirow[t]{4}{*}{24.3062090} & $\mathrm{Mn}$ & 24.374 & 24.8274137 & 25.4167 & 25.644893 & 24.6534 & 24.4442634 \\
\hline & w & 24.642 & 25.9281175 & 26.4223 & 27.056295 & 25.1347 & 25.9380345 \\
\hline & sd & $6.6 \mathrm{E}-02$ & $3.2 \mathrm{E}-01$ & 4.63E-01 & $6.67 \mathrm{E}-01$ & 2.19E-01 & $8.2 \mathrm{E}-01$ \\
\hline & FR & $100 \%$ & $100 \%$ & $100 \%$ & $100 \%$ & $100 \%$ & $100 \%$ \\
\hline \multirow{5}{*}{$\begin{array}{c}\text { G08 } \\
-0.0958250\end{array}$} & $\mathrm{~b}$ & -0.095825 & -0.0958250 & -0.095825 & -0.095825 & -0.095825 & -0.0958250 \\
\hline & Mn & -0.095825 & -0.0958250 & -0.095825 & -0.095825 & -0.095825 & -0.0958250 \\
\hline & w & -0.095825 & -0.0958250 & -0.095825 & -0.095825 & -0.095825 & -0.0958250 \\
\hline & $\mathrm{sd}$ & $2.6 \mathrm{E}-17$ & $0.0 \mathrm{E}+00$ & $0.0 \mathrm{E}+00$ & $0.0 \mathrm{E}+00$ & $0.0 \mathrm{E}+00$ & $3.8 \mathrm{E}-14$ \\
\hline & FR & $100 \%$ & $100 \%$ & $100 \%$ & $100 \%$ & $100 \%$ & $100 \%$ \\
\hline \multirow{5}{*}{$\begin{array}{c}\text { G09 } \\
680.6300573\end{array}$} & $\mathrm{~b}$ & 680.630 & 680.6801414 & 680.6309 & 680.6519 & 680.63 & 680.6300568 \\
\hline & $\mathrm{Mn}$ & 680.656 & 680.9005951 & 680.6521 & 680.8343 & 680.65 & 680.7012904 \\
\hline & w & 680.763 & 681.2122556 & 680.6965 & 681.1475 & 680.70 & 680.7253848 \\
\hline & $\mathrm{sd}$ & $3.4 \mathrm{E}-02$ & $1.3 \mathrm{E}-01$ & $1.76 \mathrm{E}-02$ & $1.34 \mathrm{E}-01$ & $1.67 \mathrm{E}-02$ & $1.2 \mathrm{E}-01$ \\
\hline & FR & $100 \%$ & $100 \%$ & $100 \%$ & $100 \%$ & $100 \%$ & $100 \%$ \\
\hline \multirow{5}{*}{$\begin{array}{c}\text { G10 } \\
7049.248020\end{array}$} & $\mathrm{~b}$ & 7054.316 & 7053.5055 & 7127.9502 & 7058.45 & 7050.8342 & 7052.603348 \\
\hline & $\mathrm{Mn}$ & 7559.192 & 7361.5796 & 8453.7902 & 8344.69 & 8020.7551 & 7451.358134 \\
\hline & w & 8835.655 & 8701.1528 & 12155.1358 & 15787.89 & 9054.2923 & 8727.057749 \\
\hline & sd & $3.4 \mathrm{E}+02$ & $2.69 \mathrm{E}+02$ & $1.23 \mathrm{E}+03$ & $1.79 \mathrm{E}+03$ & $6.21 \mathrm{E}+02$ & $4.5 \mathrm{E}+02$ \\
\hline & FR & $100 \%$ & $100 \%$ & $100 \%$ & $100 \%$ & $100 \%$ & $100 \%$ \\
\hline \multirow{5}{*}{$\begin{array}{c}\text { G11 } \\
0.7499\end{array}$} & $\mathrm{~b}$ & 0.750 & 0.74990 & 0.75 & 0.7499 & 0.7499 & 0.74990 \\
\hline & $\mathrm{Mn}$ & 0.750 & 0.75088 & 0.75 & 0.7499 & 0.7499 & 0.74990 \\
\hline & w & 0.750 & 0.77098 & 0.75 & 0.7499 & 0.7499 & 0.74990 \\
\hline & $\mathrm{sd}$ & $8.0 \mathrm{E}-05$ & $3.90 \mathrm{E}-003$ & $0.0 \mathrm{E}+00$ & $1.0 \mathrm{E}-06$ & $0.0 \mathrm{E}+00$ & $1.4 \mathrm{E}-08$ \\
\hline & FR & $100 \%$ & $100 \%$ & $100 \%$ & $100 \%$ & $100 \%$ & $100 \%$ \\
\hline \multirow{5}{*}{$\begin{array}{c}\text { G12 } \\
-1\end{array}$} & $\mathrm{~b}$ & -1.0000 & -1.0000 & -1.0000 & -1.0000 & -1.0000 & -1.0000 \\
\hline & $\mathrm{Mn}$ & -1.0000 & -1.0000 & -1.0000 & -1.0000 & -1.0000 & -1.0000 \\
\hline & w & -1.0000 & -1.0000 & -1.0000 & -1.0000 & -1.0000 & -1.0000 \\
\hline & sd & $0.0 \mathrm{E}+00$ & $0.0 \mathrm{E}+00$ & $0.0 \mathrm{E}+00$ & $0.0 \mathrm{E}+00$ & $0.0 \mathrm{E}+00$ & $1.2 \mathrm{E}-10$ \\
\hline & FR & $100 \%$ & $100 \%$ & $100 \%$ & $100 \%$ & $100 \%$ & $100 \%$ \\
\hline \multirow{5}{*}{$\begin{array}{c}\text { G13 } \\
0.0539415\end{array}$} & $\mathrm{~b}$ & 0.053957 & 0.5148793 & 0.05466 & 0.05820 & 0.054638 & $\mathbf{0 . 0 5 3 9 3 8 5 2 0}$ \\
\hline & $\mathrm{Mn}$ & 0.067543 & 1.2220168 & 0.45782 & 1.37142 & 0.458857 & 0.054025374 \\
\hline & w & 0.216915 & 4.8769912 & 1.49449 & 16.43139 & 0.994983 & 0.054212043 \\
\hline & $\mathrm{sd}$ & $3.1 \mathrm{E}-02$ & $1.14 \mathrm{E}+00$ & 3.7E-01 & $2.9 \mathrm{E}+00$ & $3.44 \mathrm{E}-01$ & $7.8 \mathrm{E}-05$ \\
\hline & FR & $100 \%$ & $24 \%$ & $100 \%$ & $100 \%$ & $100 \%$ & $100 \%$ \\
\hline
\end{tabular}

